\title{
Modeling the Frequency of Phrasal Verbs with Search Engines
}

\author{
Grażyna Chamielec \\ SuperMemo \\ Poznan, Poland \\ ika.chamielec@gmail.com
}

\author{
Dawid Weiss \\ Institute of Computer Science \\ Poznan University of Technology \\ Poznan, Poland \\ dawid.weiss@es.put.poznan.pl
}

\begin{abstract}
There are well over a thousand phrasal verbs in English. For non-native speakers they are notoriously difficult to remember and use in the right context. In this paper we present a ranking of phrasal verbs based on their estimated frequency of use. We considered the public indexable Web to be our reference corpora and used major Web search engines to acquire phraseoccurrence statistics. While this technique relies on inaccurate and possibly biased estimation functions, we show that the overall distribution of ranks seems to be consistent among all the queried search engines operated by different vendors.
\end{abstract}

Institute of Computer Science, Poznan University of Technology Research report: RA-05/08

\section{INTRODUCTION}

A phrasal verb is, according to the Cambridge Advanced Learner's Dictionary:

[...] a phrase which consists of a verb in combination with a preposition or adverb or both, the meaning of which is different from the meaning of its separate parts.

Phrasal verbs are very common in both spoken and written English ([1] lists over 6000 entries). As the definition states, the meaning of a phrasal verb cannot be guessed from individual components-many non-native users of English must therefore memorize phrasal verbs in order to be able to understand and use them in the right context. Our motivation for this work was a direct consequence of this observation.

SuperMemo ${ }^{1}$ is a company specializing in helping people learn fast, use memory efficiently and aid in self-improvement processes. SuperMemo's line of products include, among others, dictionaries and language courses. While working on a list of English phrasal verbs, we came across the following question:

- Which phrasal verbs should be memorized first?

The two other questions that stem from this one are:

- Are there any phrasal verbs that are hardly ever present in a large corpora of written language?

- Are there groups of 'frequent' and 'infrequent' phrasal verbs and is it possible to distinguish these groups?

There is certainly no definite answer to these questions. Phrasal verbs and their meaning will vary by region and

${ }^{1}$ http://www.supermemo.com dialect of English, for example. Our intention was to rely on a large corpora of existing texts rather than book resources or dictionaries. In other words, we wanted to measure possibly 'real', average case of frequency of use of each phrasal verb, and then sort them in the order of this estimated frequency.

\section{RELATED WORK}

There is a number of dictionaries [1], [2], books and papers concerning phrasal verbs and verb-particle associations at the linguistic layer. There are also on-line resources listing phrasal verbs and providing their meanings. However, we failed to find any resource that would attempt to quantitatively measure the frequency of use of phrasal verbs. The paper by Timothy Baldwin and Aline Villavicencio came closest to our expectations [3]. In this work, authors used a HPSG grammar and processed several text corpora, measuring verbparticle occurrences very accurately. While we agree that exact methods and shallow parsing should result in the best possible accuracy, we think that processing a large enough corpora of texts with less complex (and accurate) methods should yield similar results. This assumption seems to be consistent with recent trends in computational linguistics, data mining and information retrieval.

\section{PRoposed METHOdOLOGY}

We decided that it would be great if we could utilize the largest, unrestricted, text corpora existing on Earth: the Web.

Every search engine returns an estimation of the number of documents 'matching' a given query. Figure 1 illustrates a query results page with the rough number of documents matching the exact phrase 'ask out'. While the returned number is merely an estimate and may be inaccurate (see Section V), we assumed that it is correct at least to the order of magnitude, thus properly dividing frequent and relatively infrequent phrasal verbs.

\section{Phrasal Verbs CONSIDERED}

We used a hand-crafted set of phrasal verbs (PV) collected from several on-line resources and books. We made an explicit distinction between separable and inseparable PVs where it was appropriate. To every phrasal we assigned a number of different forms in which it could possibly appear in the text. Figure 2 illustrates the format of the input file, a few 


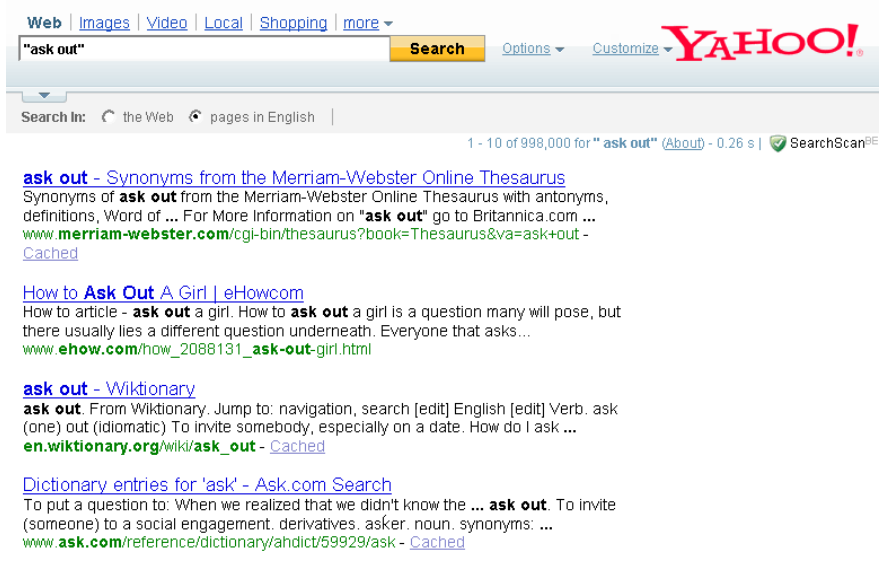

Fig. 1. Query results page from Yahoo search engine. The red rectangle marks a status line containing the number documents matching the query.

example phrasal verbs and their forms. Every line corresponds to a single phrasal verb. Various acceptable verb forms are separated by slashes. Finally, separable phrasal verbs contain an asterisk character at the word position where extra words may occur.

From this representation we could dynamically create many patterns in which a phrasal verb could appear. These patterns were then directly used to query search engines for their frequency of on-line use.

\section{Potential AMbiguities AND OTHER PROBlems}

There are a few corner cases in counting the number of documents containing a given phrasal verb and they are all a consequence of how text information retrieval methods (search engines) work. Search engines transform a document into a vector of individual words and their weights (relative importance of a given word to the document). A query to the search engine returns all documents that contain a union of the query's set of words (possibly ordered), but it is rarely possible to specify deeper contextual constraints. Let us explain the possible side-effects of this process on a few examples.

The first problem is that not every word pattern corresponds to an actual phrasal verb. For example, [to] be in can appear as I'm in, but the sole appearance of this sequence of words without the knowledge of the context may be a false result (I'm in Poland right now.). Note that this will be the case with most verbs that have transitive and intransitive forms. Another

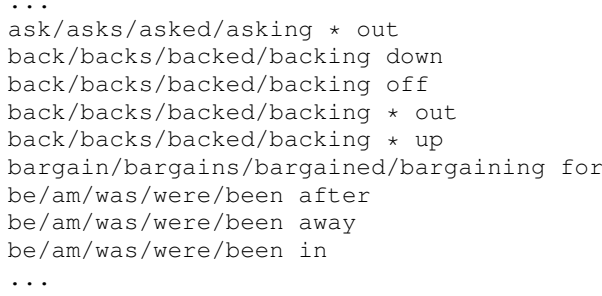

Fig. 2. An excerpt from the input list of phrasal verbs.

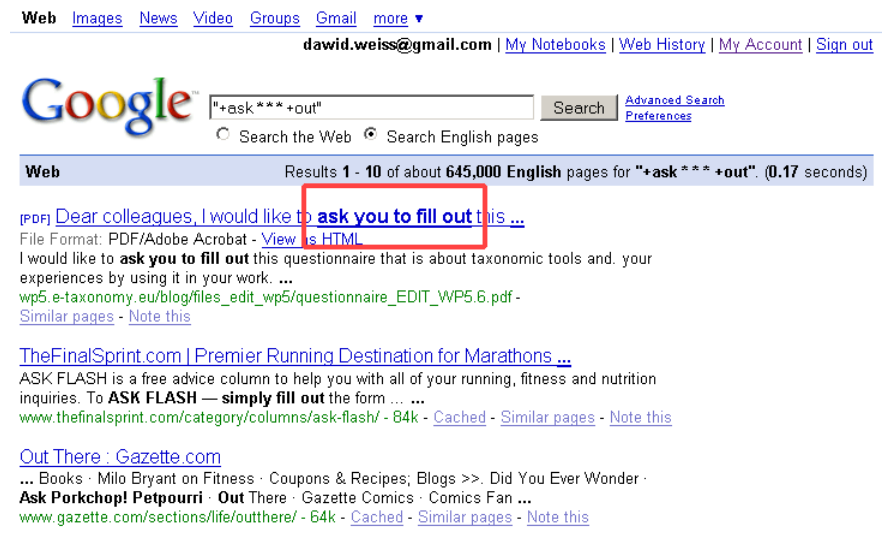

Fig. 3. A wildcard query may result in a false match (see the marked phrase).

issue is caused by multiple meanings of a single phrasal verb, compare throw up (vomit) and throw up (an idea). Detecting and separating the meaning of these two expressions seems impossible with contemporary major search engines.

The final example concerns separable forms of phrasal verbs. What we intend to do is query for patterns (sequences of words) that have a few words in between (but not too many). For example, a phrasal verb sign in can appear as sign me in, please. Without linguistic analysis, allowing too many words between components of a phrasal verb may result in many false matches. For instance, as Figure 3 illustrates, the three top-ranked documents for a query ask out separated by three other words, are basically noise.

We decided to calculate occurrence statistics regardless of the above problems. It is our assumption that the number of false matches for less than three wildcards can be neglected compared to the number of true matches. As for phrasal verbs with multiple meanings their occurrence counts all sum up to one figure which reflects their aggregated use. Since so, the final ranking position is to some extent indicative of the need to learn a given phrasal verb (even if it has multiple meanings).

\section{COLleCting OCCURRENCE STATISTICS}

We collected occurrence statistics from several search engines: Google (www.google.com), Yahoo (www.yahoo.com), AllTheWeb (www.alltheweb.com), Gigablast (www.gigablast.com) and Microsoft Live (www.live.com). Google, Yahoo and AllTheWeb support the so-called wildcard queries, i.e., a query for all documents containing a given phrase separated by one or more unrestricted words inside. With wildcard queries we could estimate the number of occurrences of separable phrasal verbs by querying for the exact phrase, phrase with one, two and three extra words at the point of possible separation. For example, the entry (to simplify, we only show one verb form here):

ask * out

would result in the following queries to a search engine:

ask out

ask * out 
\# form id; pvid; pv; form; wildcards; engine; documents $0 ; 1$; act/acts/acting/acted like; act like; 0 ;All TheWeb; 35700000 $0 ; 1$; act/acts/acting/acted like; act like;0; Gigablast;1049000 $0 ; 1 ;$ act/acts/acting/acted like; act like;0; Google; 726000 $0 ; 1 ;$ act/acts/acting/acted like; act like; 0 ; Live; 9290000 $0 ; 1 ;$ act/acts/acting/acted like; act like;0; Yahoo; 40500000

9;3; add/adds/adding/added * up; add * up; 1 ;All TheWeb;5480000 9; 3 ; add/adds/adding/added * up; add * up; 1 ; Google; 247000 9;3; add/adds/adding/added * up;add * up; $1 ;$ Yahoo;6040000

Fig. 4. A fragment of the output file with collected document counts. The meaning of fields is (from left to right, separated by semicolons): form idunique identifier of a phrasal verb's form, pv_id-unique identifier of a phrasal verb, pv-phrasal verb (as in the input), form-the query (pv's form), wildcards-number of wildcards in the query, engine-search engine, documents - estimated number of matching Web documents.

ask * * out

ask ** * out

An exact format of queries submitted to each search engine varied depending on the service provider's syntax. We found out that the query syntax and parameter details are quite crucial to the result because search engines employ various optimisations and query expansion techniques that, in our case, distorted the output. The returned estimation counts also proved to have some significant variance within the same query (a search engine would return a very different document count for consecutive executions of an identical query). We created 10 identical query lists, randomized their order and executed all queries at different times and from different machines. Finally, we paid particular attention to restricting the search to documents in the English language and to searching within document content only (exclude links pointing to the page).

The process of querying search engines was partially automated and performed in accordance with search engines policies and terms of use specifications (timeouts between queries, use of automated programming interfaces when possible).

\section{RESULTS}

Overall we collected frequency counts for 10633 various separable and inseparable forms of 991 phrasal verbs (some of these were closely related, like blend in and blend into).

The full list of results is a plain text file where each row contains the estimated number of documents from one search engine for one form of a phrasal verb. Figure 4 contains an excerpt of one of the output lists (recall that there were ten identical, independently queried lists) and explains the meaning of each column in the output.

\section{A. Differences between search engines}

Every search engine is a bit different-these differences usually concern the number of indexed documents, ranking algorithms and technical aspects of estimating the number of matching documents. Our first step was to cross-compare the numbers returned from various search engines to see if they share similar distribution and what shape this distribution is.

We took one sample list out of the ten made and for each queried phrasal verb form we grouped document counts from at least three search engines (Google, Yahoo, AllTheWeb); additional search engines-Microsoft Live and Gigablastwere available for forms with no wildcards. We then sorted all forms according to the number of documents returned by Yahoo and plotted all document counts on the vertical axis.

The overall distribution shape for all search engines is for the most part exponential-Figures 5-8 show all other search engines compared to Yahoo's distribution (vertical axes logarithmic). Exponential distribution confirms our initial intuition that a small number of phrasal verbs occurs frequently and a great deal of them are relatively infrequent in our corpus (the Web). The observed differences in distribution of document counts between different search engines are of particular interest, since (theoretically) if we consider the Web to be a global population of documents, then the index of each search engine is basically a random sample taken from this population. If so, the average count of documents between two search engines should be linearly proportional to the degree of a constant multiplier. Another way to put it is that the ordering of phrasal verb forms imposed by all search engines should be very similar between search engines.

A look at Figures 5-8 reveals that several search engines returned highly correlated results. For example, Yahoo and AllTheWeb's results are almost identical (Figure 5). This is actually something we expected because AllTheWeb's index is powered by Yahoo and minor differences may be a result of different search query routing inside Yahoo's infrastructure. There is also an evident high similarity between Yahoo, Gigablast and Microsoft Live's results (see log-log plots in Figure 12 and 13), although Microsoft and Gigablast have an order of magnitude smaller index. The only significantly different engine is Google-not only has it fewer documents compared to Yahoo, but also its distribution is strikingly different compared to other search engines. Narrowed to only non-wildcard forms, the distribution difference is even more strange because it shows two different 'traces' of frequency distribution in the area of more frequent phrasal verbs (see Figure 9, 10 and 11).

We initially thought this difference in Google's case might be caused by the fact that it has the largest infrastructure and queries may be routed to separate index sections, leading to different estimated count of results. We took a closer look at all ten samples for each query, calculating minimum, maximum, median and a truncated average (average of 6 samples after sorting and removing two minimum and maximum outliers). The outcome of this analysis is that, again, Google has the largest variation between estimated result count for a single query. As Figure 14 shows, in case of Yahoo the difference between minimum and maximum number of results is relatively small, usually the same. Microsoft Live returns a fairly consistent range of difference-usually in the order of magnitude-with the truncated average usually equal to the maximum. For Google, the difference between min and max is again the order of magnitude, but the average is less predictable and is usually in between min and max (see Figure 15 illustrating this relationship). 


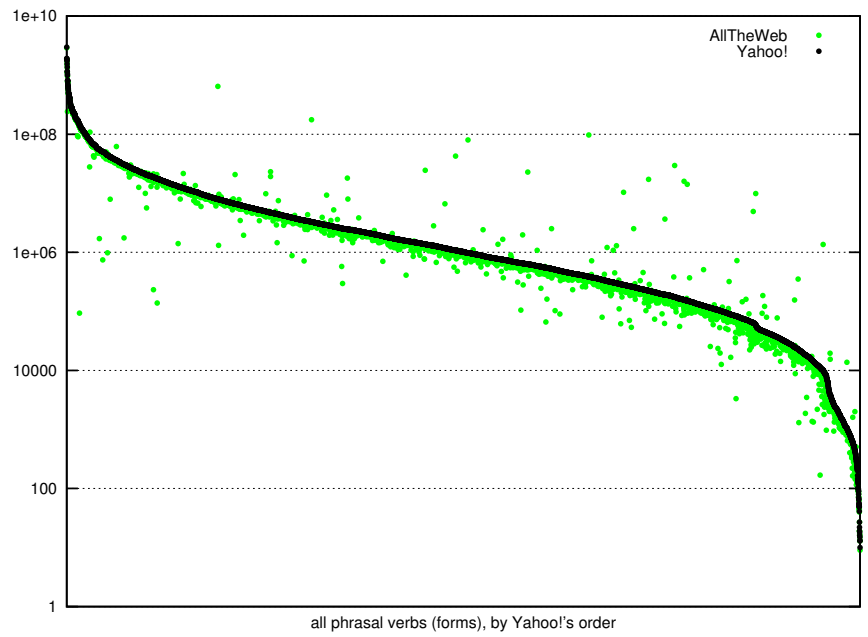

Fig. 5. Document counts for results acquired from AllTheWeb and Yahoo (sorted by Yahoo's results-the black line).

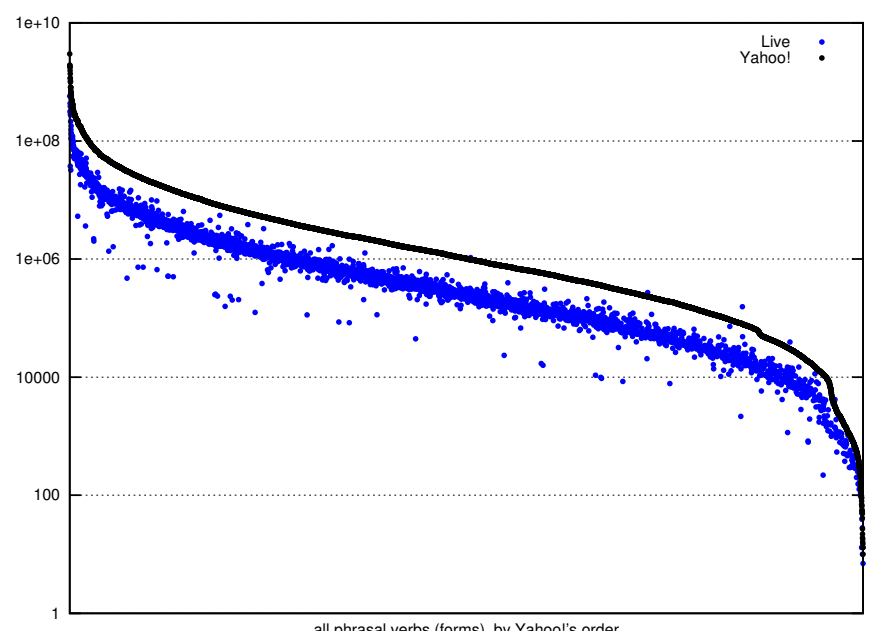

Fig. 7. Document counts for results acquired from Microsoft Live and Yahoo (sorted by Yahoo's results-the black line).

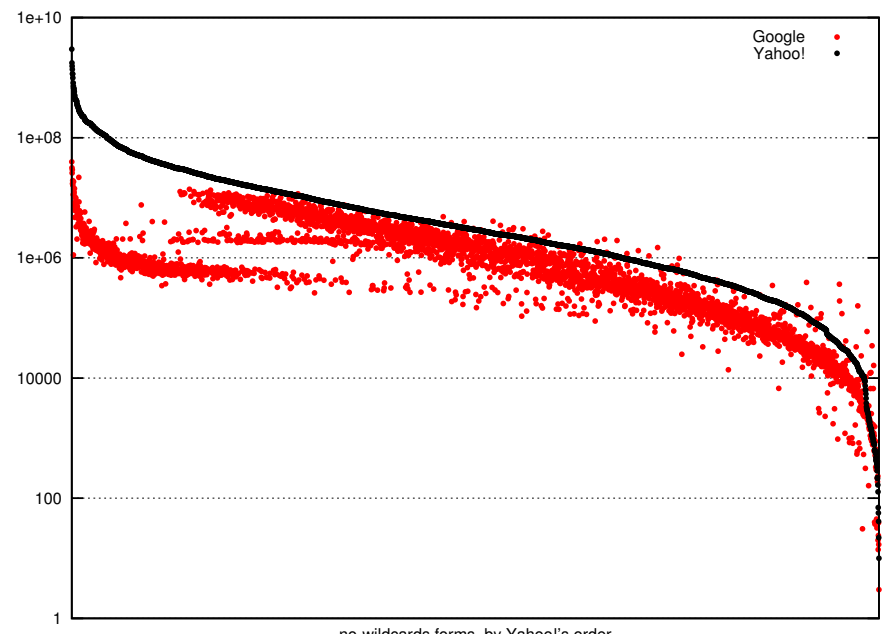

Fig. 9. Document counts for no-wildcard forms acquired from Yahoo and Google (sorted by Yahoo's results-the black line).

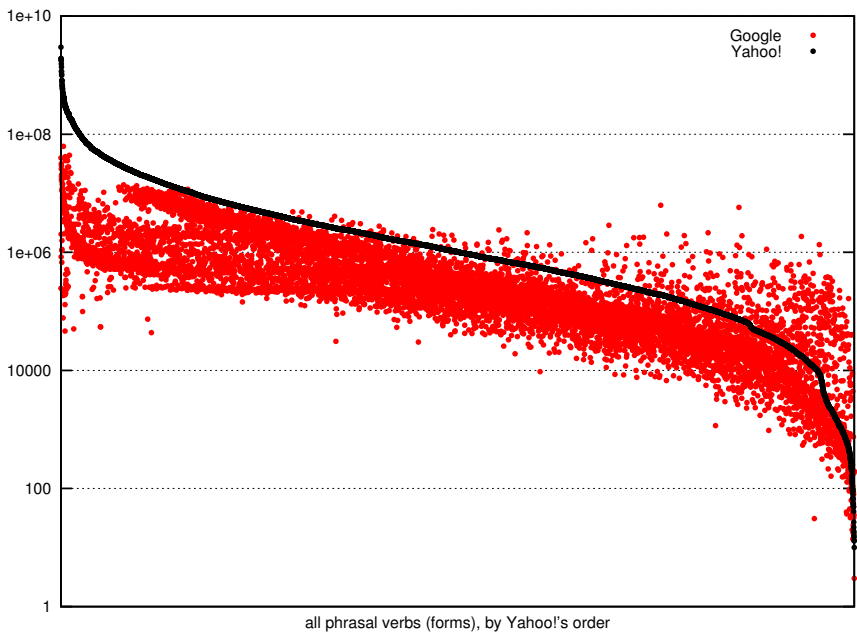

Fig. 6. Document counts for results acquired from Google and Yahoo (sorted by Yahoo's results-the black line).

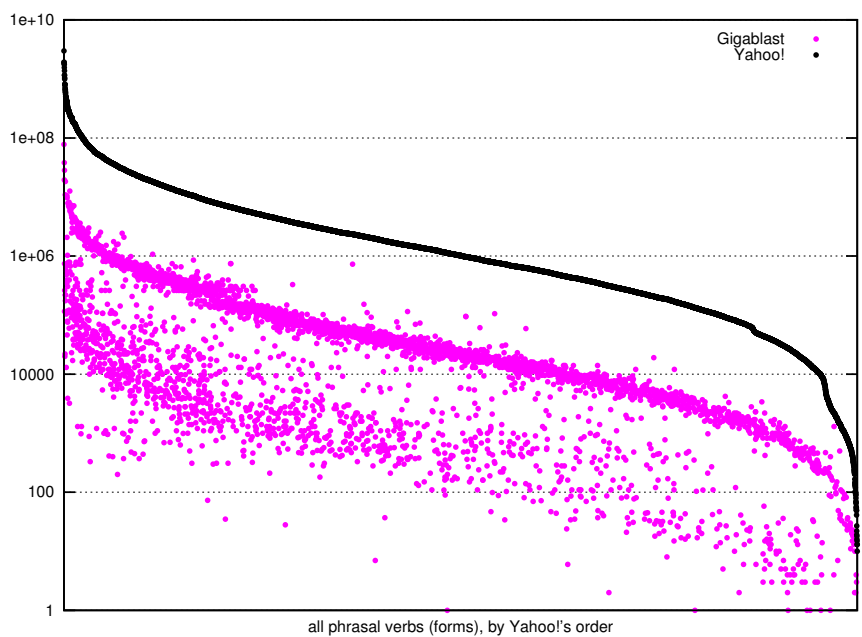

Fig. 8. Document counts for results acquired from Gigablast and Yahoo (sorted by Yahoo's results-the black line).

\section{B. Phrasal verb rankings (groups)}

In the final step of our analysis, we produced a ranking of phrasal verbs according to their totalled frequency of occurrence on the Web. Note that actual positions in this ranking are a product of multiple heuristics and their values should not be compared directly. The overall ordering should help to distinguish sub-groups of frequent and infrequent phrasal verbs and this was our initial motivation.

We experimented with many different ways of aggregating information from all samples and form of each phrasal verb. We produced multiple possible rankings based on the following algorithm:

1) for every search engine, aggregate all samples for each phrasal verb form form_id, calculate minimum, maximum, median and truncated average (avg2) from document counts;

2) consider all variations: forms with $<=0,1,2$ and 3 wildcards: 


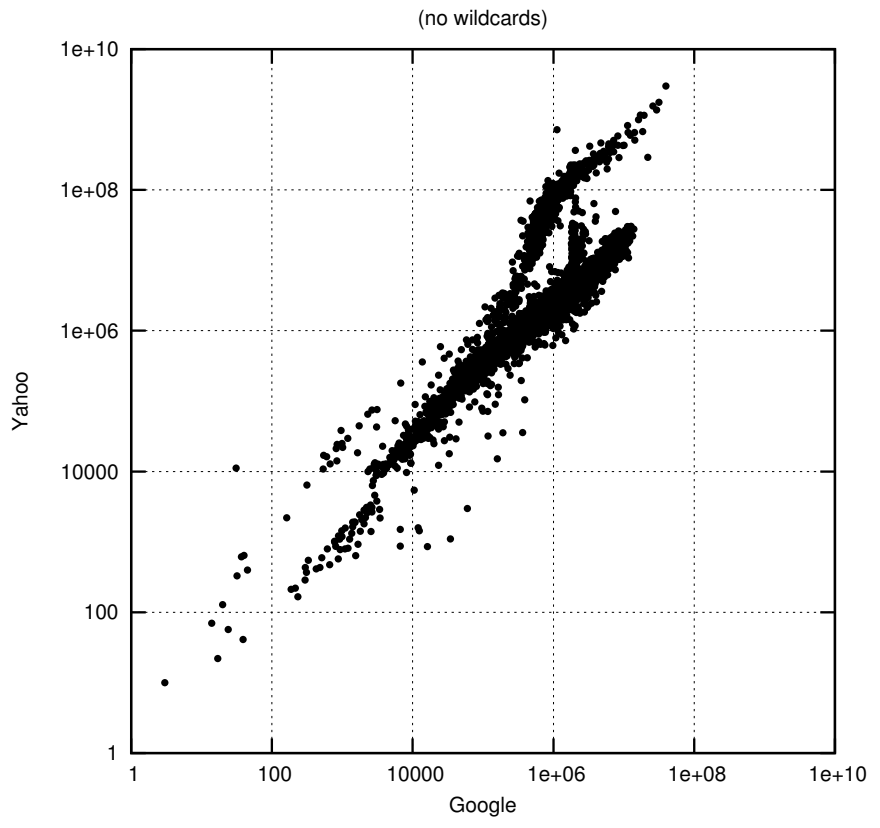

Fig. 10. Log-log plot of document counts between Yahoo and Google (no wildcards).

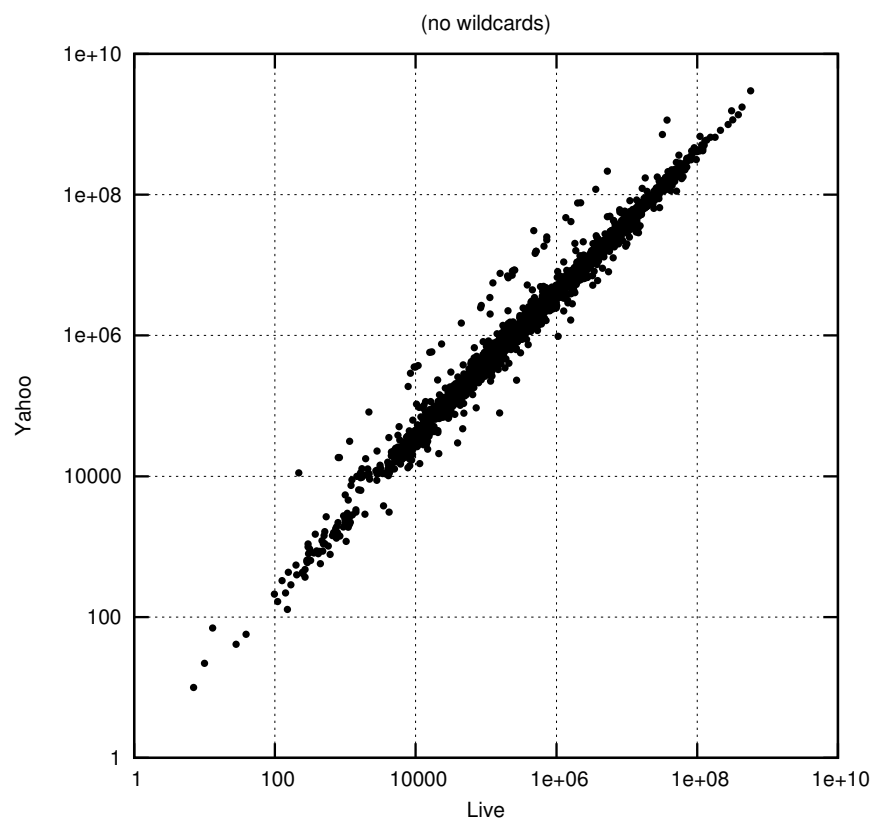

Fig. 12. Log-log plot of document counts between Yahoo and Microsoft Live (with wildcards).

3) sort in descending order all forms according to minimum, maximum, median and avg2 column, assign a rank to each form_id;

4) assign a minimum rank of any of its forms to each phrasal verb pv_id.

The above procedure results in numerous possible variations of output rankings (depending on the engine, number of wildcards and the order column being considered). These rankings, consistently with our previous analysis, demonstrate

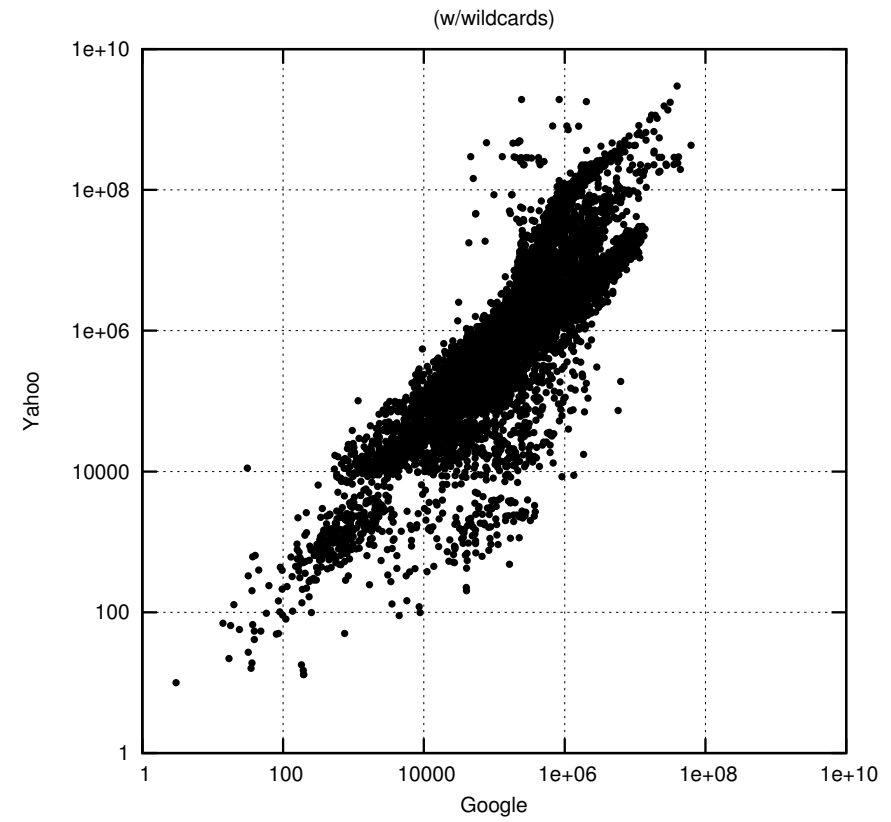

Fig. 11. Log-log plot of document counts between Yahoo and Google (with wildcards).

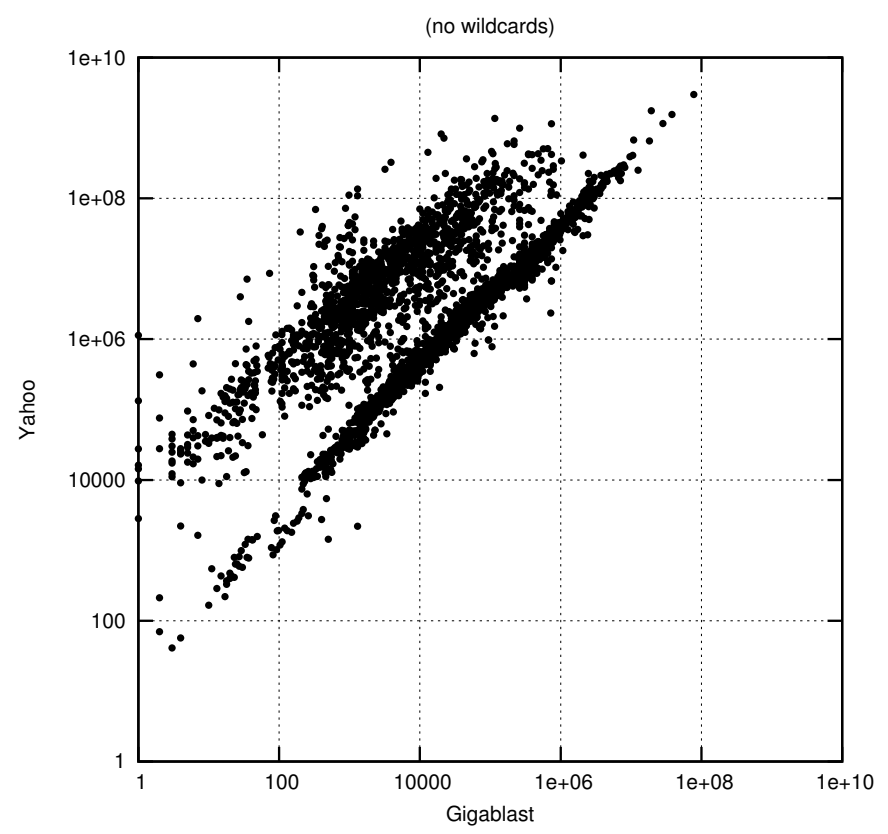

Fig. 13. Log-log plot of document counts between Yahoo and Gigablast (no wildcards).

close similarity to each other within a single search engine and between Yahoo, AllTheWeb and Microsoft Live. Only Google is an exception. To give a few examples, the choice of the sorting column did not have much impact on the actual ranking within a single search engine-Figure 16 illustrates this relationship for Google. A comparison of rankings made for forms with zero or more wildcards again shows no major differences. Cross-engine ranking consistency is shown in Figure 17. The correlation of ranks between AllTheWeb, Yahoo 

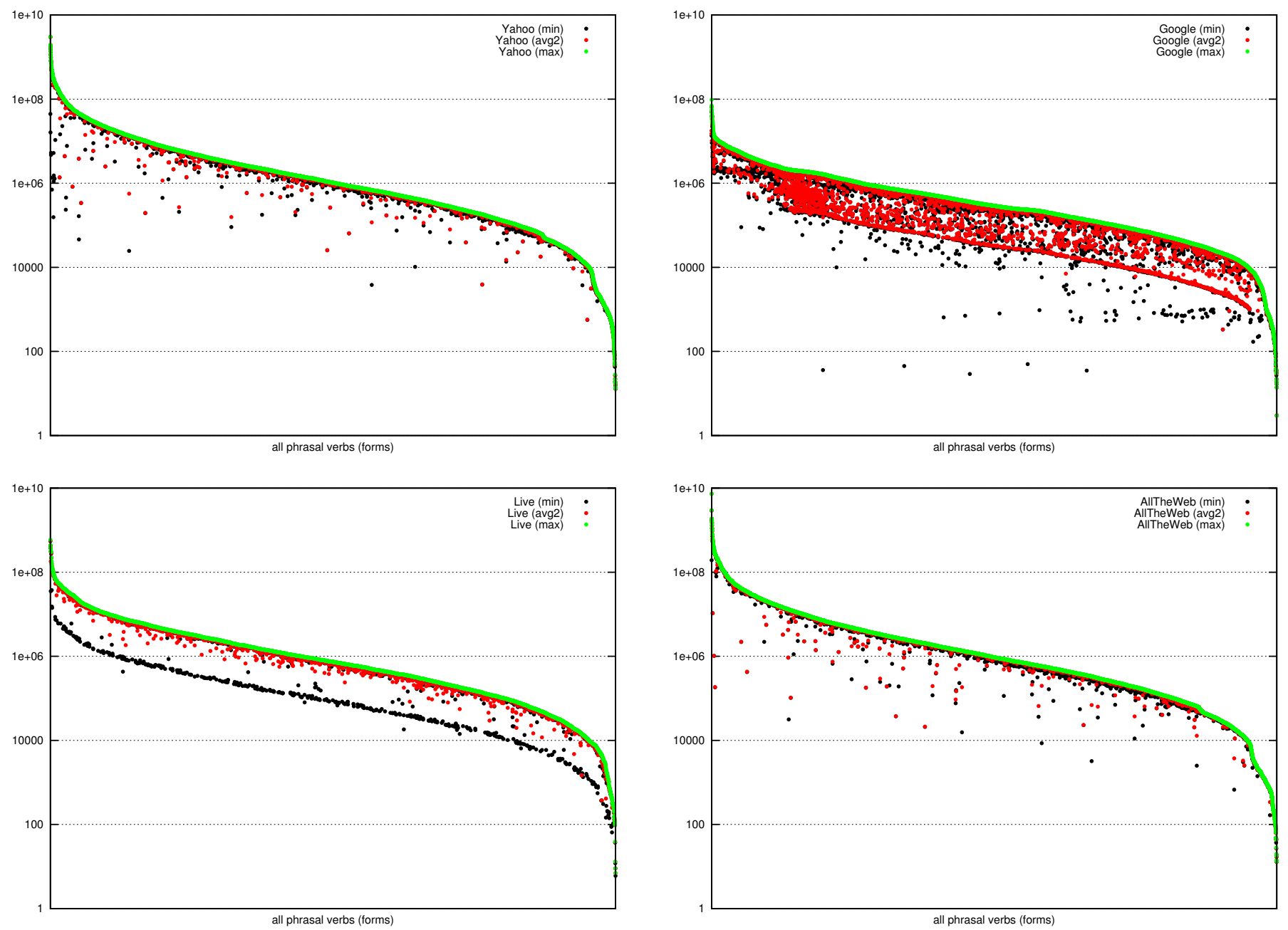

Fig. 14. Minimum and maximum number of results out of 10 samples for each phrasal verb form, horizontal axis contains all forms sorted by maximum returned results count (Yahoo, Google, Microsoft Live and AllTheWeb).

and Microsoft Live is evident (with correlation coefficient, in this case equal to Spearman's rank coefficient, larger than 0.9 for all considered combinations of rank computation). Google is clearly different (correlation coefficient with other search engines between 0.7 and 0.8 ) but we have no clear explanation as to why this is the case.

Even though all rankings were highly correlated, they were still a bit different, so there is no ultimate one answer to our initial question of 'frequent' and 'infrequent' phrasal verbs. Without a doubt the rankings themselves reflect the nature of Web resources (see Table I) by, e.g., boosting phrases common in e-commerce (sign up, check out). Yet, a tentative and subjective feeling is that the top entries are indeed something that every user of English should be familiar with and bottom ranking entries are extremely rare, uncommon or mistakes in the data set (see Table II).

Access to complete input lists of phrasal verbs, crawl results and rankings is given at the following address: http://www.cs. put.poznan.pl/dweiss/research/pv/.
TABLE I

TOP 10 PHRASAL VERBS ACCORDING TO YAHOO, GoOgLE AND Live (0 WILDCARDS, AVG 2 ).

\begin{tabular}{|c|c|c|c|}
\hline No. & Yahoo & Google & Live \\
\hline 1 & sign up & sign up & sign up \\
\hline 2 & look for & look for & look for \\
\hline 3 & check out & be in & be in \\
\hline 4 & be in & check out & check out \\
\hline 5 & look at & go back & find out \\
\hline 6 & find out & look at & look at \\
\hline 7 & arise from & find out & set up \\
\hline 8 & come to & be after & come to \\
\hline 9 & set up & look in & get to \\
\hline 10 & go back & start off & work on \\
\hline
\end{tabular}

\section{SUMMARY AND CONCLUSIONS}

We tried to create a ranking of phrasal verbs according to their frequency of actual use on the Web. We designed and performed a computational experiment, measuring estimated document count using several independent search engines. We think the outcomes are interesting in two aspects: linguistic and concerning (dis)similarities across contemporary search 

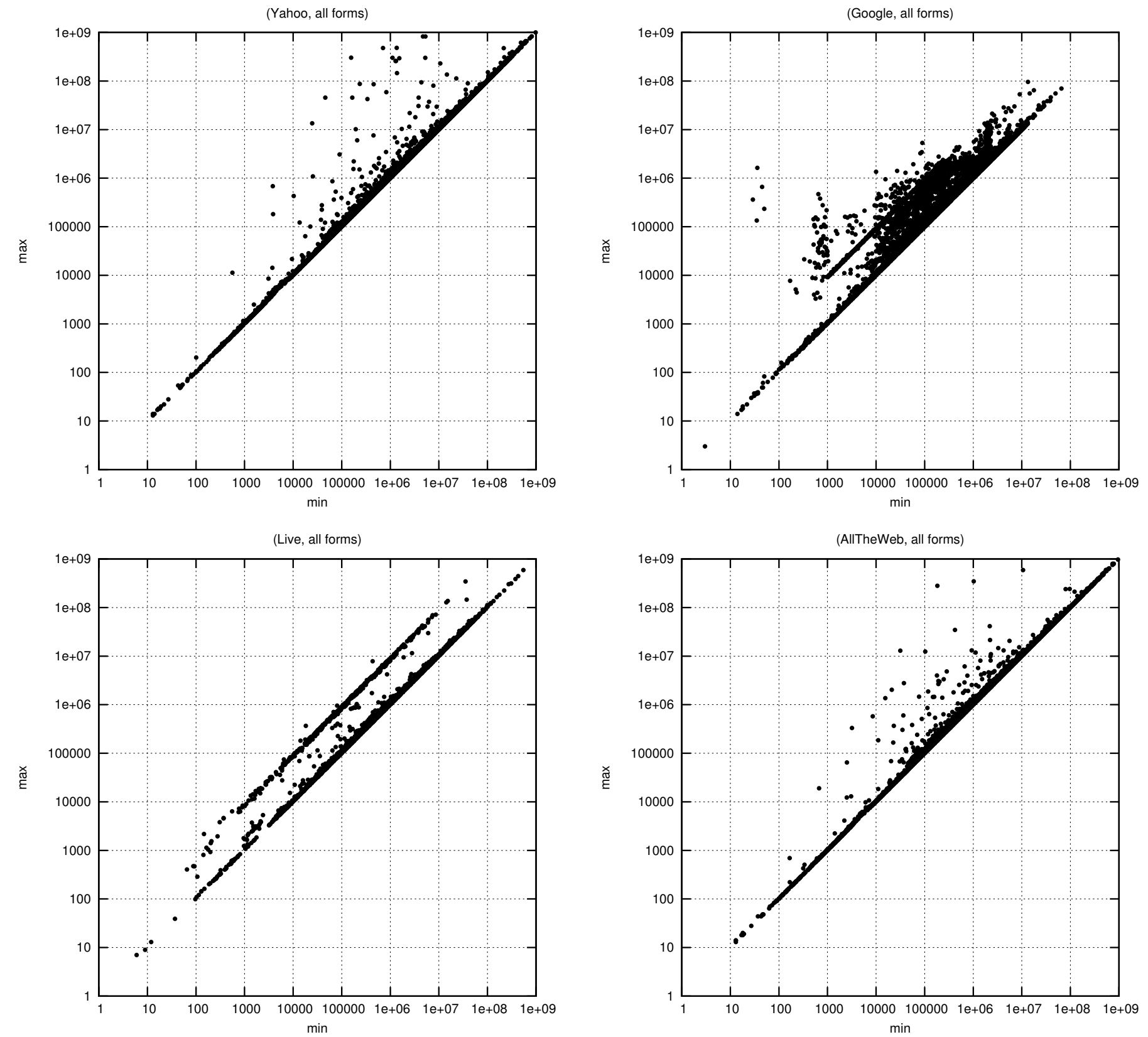

Fig. 15. Relationship between minimum and maximum number of results out of 10 samples for each phrasal verb form (Yahoo, Google, Microsoft Live and AllTheWeb).

TABLE II

SELECTED 10 PHRASAL VERBS FROM THE BOTTOM OF THE RANKING FOR YAHOO, GOOGLE AND LIVE (0 WILDCARDS, AVG2).

\begin{tabular}{ccc}
\hline Yahoo & Google & Live \\
\hline slug out & sob out & fur up \\
winkle out & slog out & sature up \\
fur up & swirl down & ravel out \\
sature up & nestle up & sponge down \\
skirt round & fur up & push round \\
sponge down & rein back & hiss off \\
tail away & skirt round & slog out \\
be bombed out & sponge down & rap put \\
slog out & ravel out & scorch along \\
hiss off & scorch along & stream down upon \\
\hline
\end{tabular}

engines.

As for the linguistic aspect, a quantitative measurement of frequenct of phrasal verbs has not been conducted before and we think there are clear indications to believe that such an analysis can yield interesting and valid results, allowing to separate frequent and infrequent phrasal verbs. A number of exciting and challenging problems remain unsolved:

- Even though the Web is very large, it also is biased; especially phrases that relate to e-commerce are boosted high up the ranking (sign up, check out).

- We currently see no way of disambiguating multi-sense phrasal verbs or no-object phrasal verbs.

- We used wildcard queries and multiple tenses for fetching 

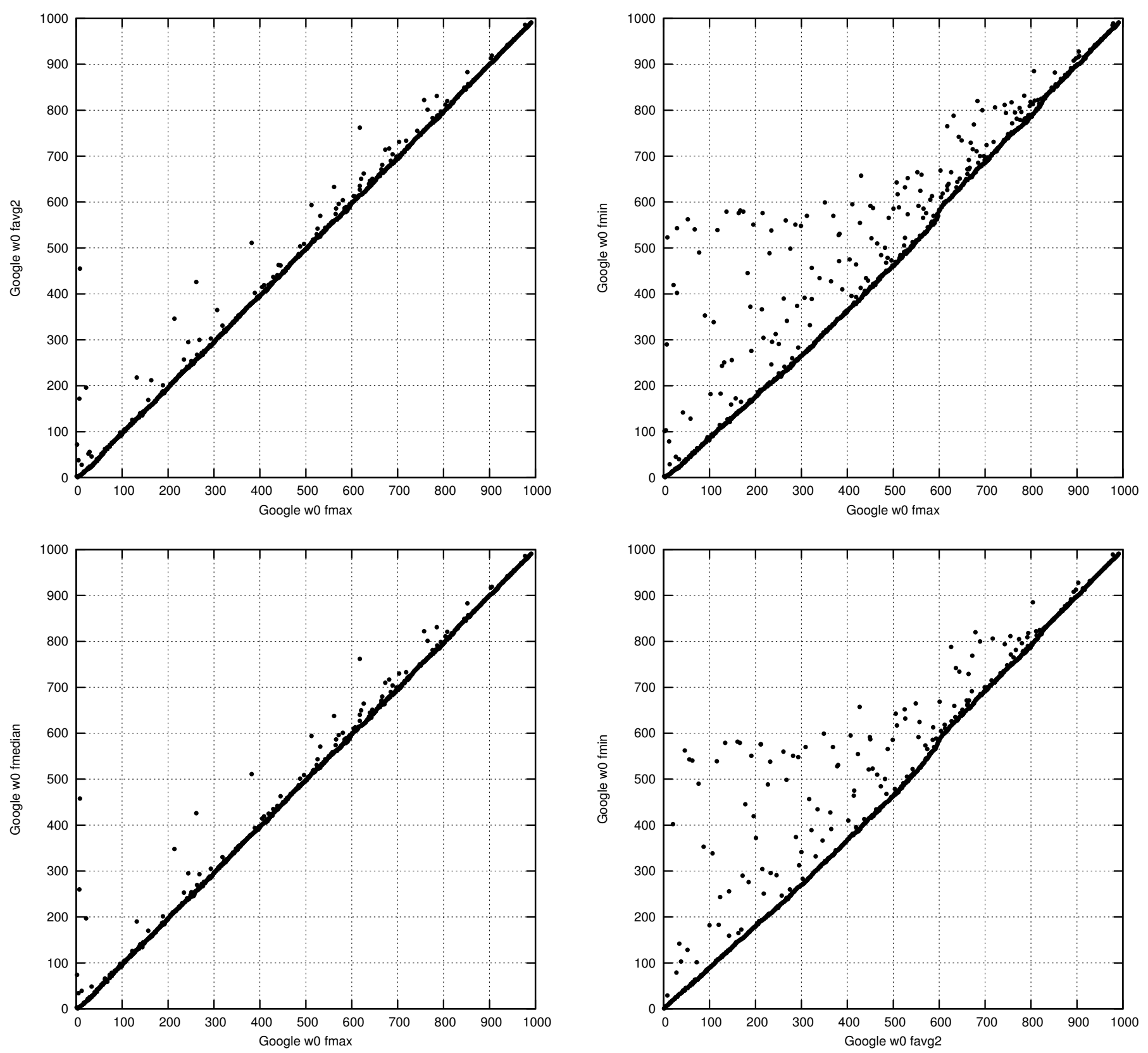

Fig. 16. Relationship between phrasal verb ranks depending on the column used for sorting forms. Google search engine, forms with zero wildcards. Axis labels contain the sorting column's abbreviation.

various potential forms of phrasal verbs, and it turned out that this had very little influence over final rankings; is such a step necessary or would it be enough to just limit the analysis to present-tense forms?

- The distribution of document counts returned from search engines is exponential, so one could make groups of phrasal verbs each falling into bins related to the frequency's order of magnitude. However, there is no clear dividing line between these bins and there is certainly some room for improvement here.

From the point of view of a researcher interested in search engines, this work provides an interesting insight into differ- ences between the search engines concerning estimation of the matching document set size.

- Yahoo is by far the most consistent search engine and its returned estimation does not vary a lot between the same queries issued at different times,

- Yahoo and Live show very correlated counts-nearly identical, in fact. This follows our intuition about 'sample from a large corpus', but is contradicted by results returned by Google. We cannot explain why Google is so much different compared to Yahoo and Live.

- Google and Live return document counts (for the same query) that vary by an order of magnitude. 

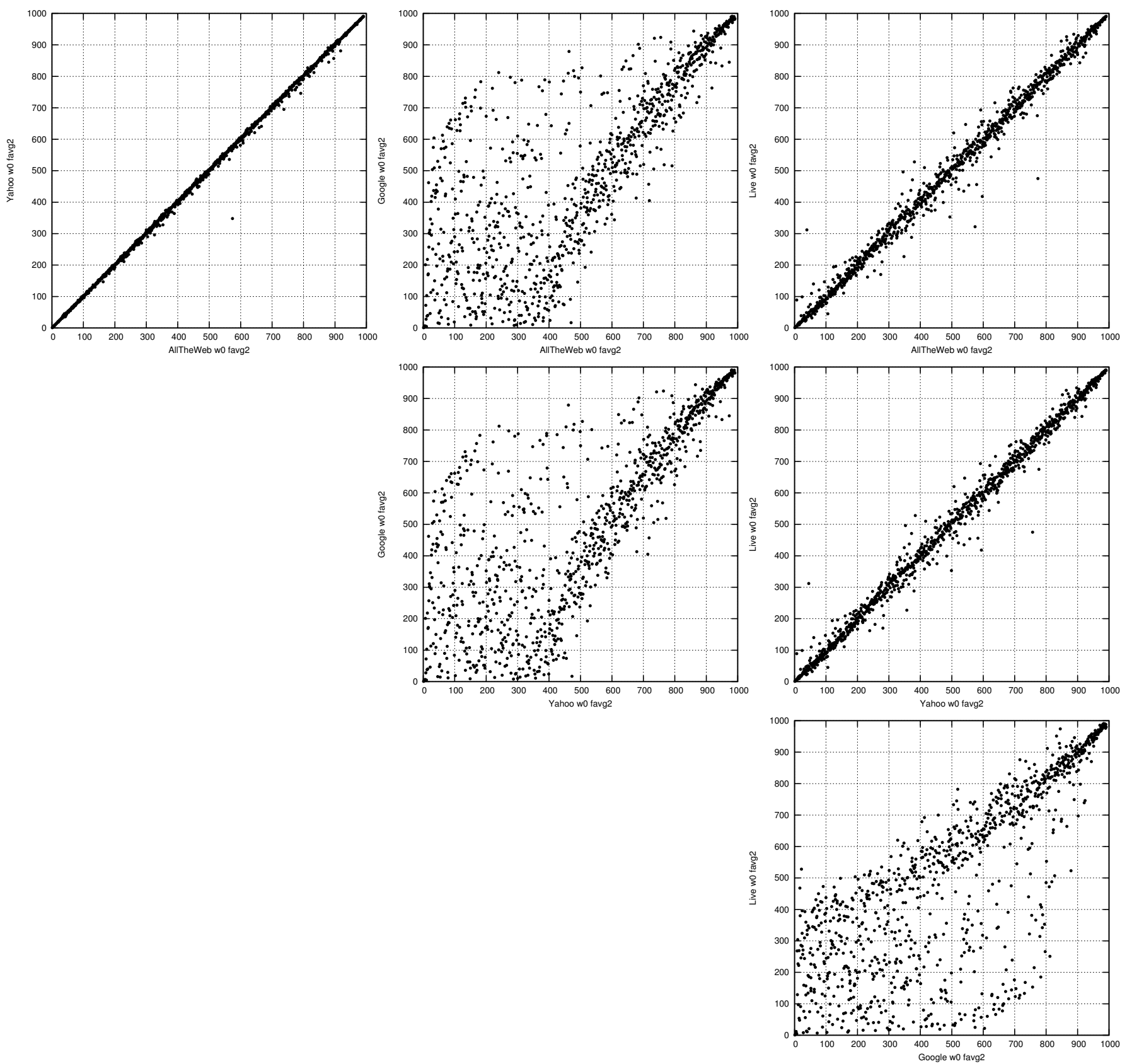

Fig. 17. Relationship between phrasal verb ranks depending on the search engine. Other parameters fixed to: avg 2 column used for sorting, zero wildcards.

As for further work on this subject, it would be quite interesting to examine phrasal verb distributions using exact NLP methods (or shallow, but with linguistic context taken into account) on a larger free corpora (such as Wikipedia or a free crawl of the Web) and compare the rankings with those we acquired from search engines. This would make it possible to validate and derive further conclusions concerning the accuracy of our method. Alternatively, one could try to estimate the number of errors made by taking the estimated number of matches by manually tagging the returned documents as false/ true matches and then establishing true/false hit ratio. This method is used successfully in software engineering to establish the true number of software defects given a number of unreliable referees assessing code quality.

\section{REFERENCES}

[1] Oxford Phrasal Verbs Dictionary for Learners of English. Oxford University Press, 2007.

[2] Cambridge Phrasal Verbs Dictionary. Cambridge University Press, 2006.

[3] T. Baldwin and A. Villavicencio, "Extracting the unextractable: a case study on verb-particles," in COLING-02: proceedings of the 6th conference on Natural language learning. Morristown, NJ, USA: Association for Computational Linguistics, 2002, pp. 98-104. 